\title{
Particle Creation in some LRS Bianchi I models
}

\author{
Luis O. Pimente** and Flavio Pineda ${ }^{\dagger}$ \\ Physics Department, Universidad Autónoma Metropolitana \\ Iztapalapa P. O. Box 55-534, 09340 México, CDMX., Мéxico
}

(Dated: March 12, 2021)

\begin{abstract}
In this work we consider particle creation by the expansion of the universe, using two Bianchi type I anisotropic models. The particles studied are of spin 0 and $1 / 2$. The cosmological models have rotational symmetry, which allows us to solve exactly the equations of motion. The number density of the created particles is calculated with the method of Bogolubov transformations.
\end{abstract}

\section{INTRODUCTION}

Quantum effects of the gravitational field are one of the greatest mysteries of nature; having a quantum theory of gravity would be one of the greatest achievements of modern science. However, we do not currently have a satisfactory theory of the quantum nature of the gravitational field that can describe and explaining the various scenarios of the very early universe. Although we do not have a satisfactory quantum gravity theory, there is no impediment to develop quantum field theory in non-flat space-time; quantum field theory can be developed including gravitational fields without a quantum theory of gravity,using a classical gravitational field, i.e. one given as a Lorentzian metric that is a solution of Einstein's equations.

The scheme is a theory describing the dynamics of quantum fields propagating in a curved spacetime background, described by a Lorentzian manifold with a general classical metric $g_{\mu \nu}$. In this way it is possible to go quite far in generalizing quantum field theory without considering the difficulties and problems involved in any quantum field theory of gravity. The Unruh effect, Hawking radiation, the production of particles in the early universe, the generation of primordial gravitational waves or even the explanation of the isotropy of the universe are some consequences of the quantum field theory in curved space-time. Particle production from vacuum is one of the most amazing predictions of quantum theory; in curved space-time, production takes place because of very intense or changing gravitational fields such as the expansion of the universe, an effect Schrödinger expected to occur [1], or the field produced by a black hole, effect studied by Hawking [2]. Parker's pioneering works [3 5 ] establishes that there is a creation of particles in the very early stages of the expansion of the universe; if this particle creation at very early times is characterized by the fundamental constants $\hbar, c, G$, then is consistent with the entropy demanded by the cosmic background radiation with a temperature of $2,7 \mathrm{~K}[6,7]$.

The majority of the works on particle production deal with the production of particles in homogeneous and isotropic universes without initial singularity in $t=0$, for example, De Sitter's universe [8], models of Friedman-Robertson-Walker [9, 10] or models of an asymptotically flat universe [11]; very few works discuss the production of particles in homogeneous anisotropic universes with initial singularity. The reason for this is partly that the usual techniques of quantum field theory in gravitational backgrounds fail when there is an initial singularity, so a different approach must be taken.

The different methods that exist to address this problem are

\footnotetext{
*Electronic address: lopr@xanum.uam.mx (Corresponding author)

${ }^{\dagger}$ Electronic address: fpineda@xanum.uam.mx
} 
1. Hamiltonian diagonalization [9, 12]

2. Feynman path-integral method [13, 14]

3. Semi-classical method [8, 15, 16]

Bukhbinder [12] develops the Hamiltonian diagonalization method and applies it to calculate the mean number of scalar particles created in a Bianchi I anisotropic universe, and the result obtained is Bose's distribution; Chitre and Hartle [13] developed the path integral method as a quantization method for a scalar field propagating in a homogeneous universe with a linear expansion, $a(t)=t$ and initial singularity in $t=0$, while Duru [14] uses this method to calculate the mean number of scalar particles in Bianchi I model

$$
\mathrm{d} s^{2}=-\mathrm{d} t^{2}+t^{2 q}\left(\mathrm{~d} x^{2}+\mathrm{d} y^{2}\right)+t^{2(1-2 q)} \mathrm{d} z^{2} .
$$

This metric is a one-parameter family of solutions to Einstein's equations with a perfect stiff fluid or a free massless scalar field as material content [17].

The semi-classical method has been used in different scenarios with favourable results, in particular, Villalba [8, 15, 16] has used it to discuss particle production in different scenarios. This method consists of solving the covariant Hamilton-Jacobi equation and compares the asymptotic behaviour of the solutions with the asymptotic behaviour of the solutions of the main field equations (Klein-Gordon equation or Dirac equation). In this paper, we discuss the production of scalar and spin $1 / 2$ particles in some Bianchi I LRS models by the semi-classical method. We present the asymptotic solutions of the field equations and compared them with the exact solutions of the Klein-Gordon (KG) and Dirac equations for massive and non-massive particles, to define the positive and negative frequency states; to solve the Dirac equation, we used the method of separation of variables developed by Villalba and Shihskin [18]. Finally, we calculated the number density of the created particles with the method of the Bogolubov transformations.

The cases that interest us are the following:

$$
\begin{aligned}
& q=0, \quad \mathrm{~d} s^{2}=-\mathrm{d} t^{2}+\mathrm{d} x^{2}+\mathrm{d} y^{2}+t^{2} \mathrm{~d} z^{2} \\
& q=1, \quad \mathrm{~d} s^{2}=-\mathrm{d} t^{2}+t^{2}\left(\mathrm{~d} x^{2}+\mathrm{d} y^{2}\right)+t^{-2} \mathrm{~d} z^{2} .
\end{aligned}
$$

The case $q=0$ represents flat space-time, which is a particular solution of the Kasner metric, while the case $q=1$ represents an expanding homogeneous universe.

\section{ASYMPTOTIC SOLUTION TO THE HAMILTON JACOBI EQUATION}

We must solve the Hamilton-Jacobi equation for the LRS Bianchi I model given by the metric (I.1). To define particles with the exact solution of the KG equation a generally covariant approach should be used, which is based on solutions of the Hamilton-Jacobi equation in the respective space-time. The way to proceed consists of the following steps [10]

1. Solve the Hamilton-Jacobi equation for the respective cosmological model.

2. Solve the KG and Dirac equations for the respective cosmological model. 
3. Compare the asymptotic behaviour of the solutions of the field equations and the HamiltonJacobi equation.

4. Positive and negative frequency states are obtained according to the following correspondence

$$
\Phi(x) \sim\left\{\begin{array}{r}
e^{-i S} \text { Positive frequency states } \\
e^{i S} \text { Negative frequency states }
\end{array}\right.
$$

where $S$ is the classical action and $\Phi$ is the wave function.

The covariant Hamilton-Jacobi equation can be written as

$$
g^{\mu \nu} \partial_{\mu} S \partial_{\nu} S+m^{2}=0
$$

Since the metric (I.1) is only dependent on time, the variables of the $S$ function can be separated as

$$
S(\mathbf{k}, \mathbf{r}, t)=\mathbf{k} \cdot \mathbf{r}+f(t) .
$$

Substituting (II.6) into (II.5) we obtain the equation for the $f$ function

$$
-\left(\frac{\mathrm{d} f}{\mathrm{~d} t}\right)^{2}+t^{-2 q} k_{\perp}^{2}+t^{2(2 q-1)} k_{z}^{2}+m^{2}=0,
$$

where $k_{\perp}^{2}=k_{x}^{2}+k_{y}^{2}$. For $q=0$, the asymptotic behavior of the solution is

$$
f_{k}(t) \sim \pm \log t^{k_{z}}
$$

as $t \rightarrow 0$, that is, in the initial singularity, and

$$
f_{k}(t) \sim \pm \sqrt{k_{\perp}^{2}+m^{2}} t
$$

as $t \rightarrow \infty$. The classic action for this Bianchi model has the following asymptotic behaviour

$$
S(\mathbf{k}, \mathbf{r}, t) \sim\left\{\begin{array}{cl}
\mathbf{k} \cdot \mathbf{r} \pm \log t^{k_{z}}, & t \rightarrow 0 \\
\mathbf{k} \cdot \mathbf{r} \pm \sqrt{k_{\perp}^{2}+m^{2}} t, \quad t \rightarrow \infty .
\end{array}\right.
$$

Therefore, the wave function $\Phi_{k}$ should have the following semiclassical behaviour

$$
\Phi_{k} \sim\left\{\begin{array}{r}
e^{i \mathbf{k} \cdot \mathbf{r}} t^{ \pm i k_{z}}, \quad t \rightarrow 0 \\
e^{i \mathbf{k} \cdot \mathbf{r}} e^{ \pm i \sqrt{k_{\perp}^{2}+m^{2}} t}, \quad t \rightarrow \infty
\end{array}\right.
$$


For the case $q=1$, the asymptotic behaviour of the solution of the Hamilton-Jacobi equation is

$$
f_{k}(t) \sim \pm \log t^{k_{\perp}}
$$

as $t \rightarrow 0$ and

$$
f_{k}(t) \sim \pm \frac{k_{z} t^{2}}{2} \pm \frac{k_{z} m^{2}}{2} \log t,
$$

as $t \rightarrow \infty$. Hence, the wave function $\Phi$ should have the following semi-classical behaviour

$$
\Phi_{k} \sim\left\{\begin{array}{r}
e^{i \mathbf{k} \cdot \mathbf{r}} t^{ \pm i k_{\perp}}, \quad t \rightarrow 0 \\
e^{i \mathbf{k} \cdot \mathbf{r}} e^{ \pm i k_{z} t^{2} / 2} t^{ \pm i k_{z} m^{2} / 2}, \quad t \rightarrow \infty .
\end{array}\right.
$$

To identify the negative and positive frequency states of scalar and spin $1 / 2$ particles it is necessary to solve the KG and Dirac equation in the Bianchi I model for cases $q=0$ and $q=1$.

\section{KLEIN GORDON EQUATION}

The massive KG equation with arbitrary coupling $\xi$ in curved space-time takes the form

$$
\left(g^{\mu \nu} \nabla_{\mu} \nabla_{\nu}-m^{2}-\xi R\right) \Phi=0
$$

where $R$ is the scalar curvature, $\xi$ is a dimensionless coupling constant, which in the case of a conformally coupled field takes the value of $\xi=1 / 6$ and for the minimal coupling case $\xi=0$, and $\nabla_{\mu}$ is the covariant derivative. For the metric (I.1), the equation is

$$
\frac{\mathrm{d}^{2} f_{k}}{\mathrm{~d} t^{2}}+\left[\frac{1}{t^{2}}\left(\frac{1}{4}+2 q \xi(3 q-2)\right)+t^{-2 q}{k_{\perp}}^{2}+t^{4 q-2} k_{z}{ }^{2}+m^{2}\right] f_{k}(t)=0,
$$

where we have separated variables of the form

$$
\Phi(x)=t^{-1 / 2} e^{i \mathbf{k} \cdot \mathbf{r}} f_{k}(t),
$$

and we have substituted the scalar curvature for the metric (I.1)

$$
R=\frac{2 q(3 q-2)}{t^{2}}
$$

Exact solutions for the cases $q=0$ and $q=1$ were obtained by Pimentel [19].

- For the case $q=0$, the KG equation takes the form

$$
\frac{\mathrm{d}^{2} f_{k}}{\mathrm{~d} t^{2}}+\left[\frac{1}{t^{2}}\left(\frac{1}{4}+k_{z}{ }^{2}\right)+{k_{\perp}}^{2}+m^{2}\right] f_{k}(t)=0
$$


and the solution is

$$
\Phi(x)=e^{i \mathrm{k} \cdot \mathrm{r}}\left[A_{1} H_{i k_{z}}^{(1)}\left(\sqrt{{k_{\perp}}^{2}+m^{2}} t\right)+B_{1} H_{i k_{z}}^{(2)}\left(\sqrt{k_{\perp}^{2}+m^{2}} t\right)\right]
$$

where $H_{\nu}^{(j)}$ is the Hankel function of order $\nu$ and $A_{1}, B_{1}$ are integration constants.

- For the case $q=1$, the KG equation takes the following form:

$$
\frac{\mathrm{d}^{2} f_{k}}{\mathrm{~d} t^{2}}+\left[\frac{1}{t^{2}}\left(\frac{1}{4}+2 \xi+k_{\perp}^{2}\right)+t^{2} k_{z}^{2}+m^{2}\right] f_{k}(t)=0
$$

and the solution is

$$
\Phi(x)=t^{-1} e^{i \mathbf{k} \cdot \mathbf{r}}\left[A_{1} M_{\kappa, \mu}\left(i k_{z} t^{2}\right)+B_{1} W_{\kappa, \mu}\left(i k_{z} t^{2}\right)\right]
$$

where $M_{\kappa \mu}, W_{\kappa \nu}$ are Whittaker's function and

$$
\kappa=-\frac{i m^{2}}{4 k_{z}}, \quad \mu=\frac{i}{2} \sqrt{k_{\perp}^{2}+2 \xi} .
$$

\section{PRODUCTION OF SCALAR PARTICLES}

To identify the positive and negative frequency states in the $t \rightarrow 0$ and $t \rightarrow \infty$, the asymptotic solutions should be compared with the solution obtained from the semiclassic method.

1. Case $q=0$.

The solution of KG's equation for $q=0$ are Hankel functions (III.20); the asymptotic behaviour of the Bessel function $J_{\nu}(z)$ for $z \rightarrow 0$ is given by the asymptotic formula [20]

$$
J_{\nu}(z) \sim \frac{z^{\nu}}{2^{\nu} \Gamma(1+\nu)}
$$

Therefore, the solution (III.20) presents the following asymptotic behaviour in $t \rightarrow 0$

$$
J_{i k_{z}}\left(\sqrt{k_{\perp}^{2}+m^{2}} t\right) \sim \frac{t^{i k_{z}}}{2^{i k_{z}} \Gamma\left(1+i k_{z}\right)} \sim t^{i k_{z}}, \quad t \rightarrow 0 .
$$

Compared to the solution of the semiclassic method (II.11), we see that the states of negative frequency are defined as

$$
f_{(0)}^{-}(t)=A_{(0)}^{-} J_{i k_{z}}\left(\sqrt{k_{\perp}^{2}+m^{2}} t\right),
$$


where $A_{(0)}^{-}$is a normalization constant. The positive frequency state $f_{(0)}^{+}(t)$ is the complex conjugate of (IV.26)

$$
f_{(0)}^{+}(t)=\left[f_{(0)}^{-}(t)\right]^{*}=A_{(0)}^{+} J_{-i k_{z}}\left(\sqrt{k_{\perp}^{2}+m^{2}} t\right) .
$$

On the other hand, the asymptotic behaviour of the Hankel function $H_{\nu}^{(2)}(z)$ for $z \rightarrow \infty$ is [20]

$$
H_{\nu}^{(2)}(z) \sim \sqrt{\frac{2}{\pi z}} \exp [-i(z-\nu \pi / 2-\pi / 4)]
$$

Therefore, the solution (III.20) presents the following asymptotic behaviour in $t \rightarrow \infty$

$$
H_{i k_{z}}^{(2)}\left(\sqrt{k_{\perp}^{2}+m^{2}} t\right) \sim t^{-1 / 2} e^{-i \sqrt{k_{\perp}^{2}+m^{2}} t} . \quad t \rightarrow \infty
$$

Compared to the semiclassical solution (II.11), this defines the positive frequency states at $t \rightarrow \infty$

$$
f_{(\infty)}^{+}(t)=B_{(\infty)}^{+} H_{i k_{z}}^{(2)}\left(\sqrt{k_{\perp}^{2}+m^{2}} t\right)
$$

Positive frequency state $f_{(\infty)}^{+}(t)$ is related to $f_{(0)}^{+}(t)$ and $f_{(0)}^{-}(t)$ by means of the Bogolubov transformations [21]

$$
f_{(\infty)}^{+}(t)=\alpha f_{(0)}^{+}(t)+\beta f_{(0)}^{-}(t)
$$

The use of the connection formula of the Bessel functions [20]

$$
H_{\nu}^{(2)}(z)=\frac{e^{i \pi \nu} J_{\nu}(z)-J_{-\nu}(z)}{i \sin \pi \nu}
$$

allows us to calculate the Bogolubov coefficients $\alpha$ and $\beta$

$$
\alpha A_{(0)}^{+}=i B_{(\infty)}^{+} \csc \left(i \pi k_{z}\right), \quad \beta A_{(0)}^{+}=-i B_{(\infty)}^{+} e^{-\pi k_{z}} \csc \left(i \pi k_{z}\right)
$$

Then

$$
\frac{|\alpha|^{2}}{|\beta|^{2}}=e^{2 \pi k_{z}}
$$

Due to the orthogonality relation, the coefficients satisfy 


$$
|\alpha|^{2}+|\beta|^{2}=1
$$

Therefore, the number density of created particles by the evolution of the cosmological model is

$$
n(k)=\left(\frac{|\alpha|^{2}}{|\beta|^{2}}-1\right)^{-1}=\left(e^{2 \pi k_{z}}-1\right)^{-1}
$$

This result, which is a Bose-Einstein distribution, coincides with the result by Duru 14] obtained with the path integral method. The dependence on the direction $k_{z}$ indicates that the created particle density is distributed in a uniform way along $k_{z}$, that is, the expansion of the universe has a privileged direction to create scalar particles.

2. Case $q=1$

In this case KG's equation solution are Whittaker functions (III.22); the asymptotic behaviour of these functions is [20]

For $z \rightarrow 0$

$$
M_{\kappa \mu}(z) \sim z^{1 / 2+\mu}, \quad W_{\kappa \mu}(z) \sim \frac{\Gamma(2 \mu)}{\Gamma(1 / 2+\mu-\kappa)} z^{1 / 2-\mu}
$$

and if $z \rightarrow \infty$

$$
M_{\kappa \mu}(z) \sim \frac{\Gamma(2 \mu+1)}{\Gamma(1 / 2+\mu-\kappa)} e^{z / 2} z^{-\kappa}, \quad W_{\kappa \mu}(z) \sim e^{-z / 2} z^{\kappa}
$$

In the limit $t \rightarrow 0$, the solution (III.22) is

$$
M_{\kappa, \mu}\left(i k_{z} t^{2}\right) \sim t^{1+i \sqrt{k_{\perp}^{2}+2 \xi}}
$$

If we compare with the asymptotic solution in $t \rightarrow 0$ by the semiclassical method we define the positive frequency states in the initial singularity as

$$
u_{(0)}^{+}(t)=A_{(0)}^{+} t^{-1} M_{\kappa \mu}\left(i k_{z} t^{2}\right)
$$

while negative frequency states are defined as the complex conjugate of (IV.40)

$$
u_{(0)}^{-}(t)=\left[u_{(0)}^{+}(t)\right]^{*}=A_{(0)}^{-} t^{-1}\left(e^{i \pi}\right)^{1 / 2-\mu} M_{\kappa,-\mu}\left(i k_{z} t^{2}\right) .
$$


The asymptotic behaviour of the scalar field of the exact solution of the KG equation in the infinite future $t \rightarrow \infty$ for the function $W_{\kappa \mu}\left(i k_{z} t^{2}\right)$ corresponds to the semiclassical solution with the minus ( - ) sign, i.e, we define the states of negative frequency in $t \rightarrow \infty$ as

$$
u_{(\infty)}^{+}(t)=B_{(\infty)}^{+} t^{-1} W_{\kappa \mu}\left(i k_{z} t^{2}\right)
$$

We use Bogolubov's transformations to relate $u_{(\infty)}^{+}(t)$ with $u_{(0)}^{+}(t), u_{(0)}^{-}(t)$

$$
u_{(\infty)}^{+}(t)=\alpha u_{(0)}^{+}(t)+\beta u_{(0)}^{-}(t)
$$

To calculate the Bogolubov coefficients, we use the Whittaker function connection formula [20]

$$
W_{\kappa \mu}\left(i k_{z} t^{2}\right)=\frac{\Gamma(-2 \mu)}{\Gamma(1 / 2-\mu-\kappa)} M_{\kappa \mu}\left(i k_{z} t^{2}\right)+\frac{\Gamma(2 \mu)}{\Gamma(1 / 2+\mu-\kappa)} M_{\kappa,-\mu}\left(i k_{z} t^{2}\right)
$$

Then

$$
\frac{|\beta|^{2}}{|\alpha|^{2}}=e^{\pi \sqrt{k_{\perp}^{2}+2 \xi}}
$$

The number density of the created particles is

$$
n(k)=\left(\frac{|\alpha|^{2}}{|\beta|^{2}}-1\right)^{-1}=\left(e^{\pi} \sqrt{k_{\perp}^{2}+2 \xi}-1\right)^{-1} .
$$

This expression shows that the density of particles created is the Bose-Einstein distribution, and coincides, once again, with the Duru [14] result obtained by the path integral method.

\section{DIRAC EQUATION}

We proceed to solve the Dirac equation in the Bianchi I model. The Dirac equation in curved space-time can be written as

$$
\left[\tilde{\gamma}^{\mu}(x)\left(\partial_{\mu}-\Gamma_{\mu}\right)+m\right] \Psi(x)=0,
$$

where $\Gamma_{\mu}$ the spin connections that are calculated through the expression [22]

$$
\Gamma_{\mu}=\frac{1}{4} \omega_{\mu \alpha \beta} \gamma^{\alpha} \gamma^{\beta},
$$

where $\omega_{\mu \alpha \beta}$ are Ricci's rotation coefficients and $e^{\mu}{ }_{\alpha}(x)$ is a tetrad that satisfies the relation 


$$
g_{\mu \nu}(x)=e_{\mu}^{\alpha} e_{\nu}^{\beta} \eta_{\alpha \beta}
$$

Dirac matrices in curved space-time are $\tilde{\gamma}^{\mu}(x)$ that are related to gamma matrices $\gamma^{\mu}$ of flat spacetime by

$$
\tilde{\gamma}^{\mu}(x)=e_{\alpha}^{\mu}(x) \gamma^{\alpha}
$$

and comply with the anticommutation rule

$$
\left\{\tilde{\gamma}^{\mu}, \tilde{\gamma}^{\nu}\right\}=2 g^{\mu \nu}
$$

The tetrad for the metric is chosen diagonally

$$
e^{0}=\mathrm{d} t, \quad e^{1}=t^{q} \mathrm{~d} x, \quad e^{2}=t^{q} \mathrm{~d} y \quad e^{3}=t^{1-2 q} \mathrm{~d} z
$$

while the spin connections are given by

$$
\Gamma_{0}=0, \quad \Gamma_{1}=\frac{1}{2} q t^{q-1} \gamma^{1} \gamma^{0}, \quad \Gamma_{2}=\frac{1}{2} q t^{q-1} \gamma^{2} \gamma^{0}, \quad \Gamma_{3}=\frac{1}{2}(1-2 q) t^{-2 q} \gamma^{3} \gamma^{0},
$$

and Dirac matrices in curved space-time are

$$
\tilde{\gamma}^{0}(x)=\gamma^{0}, \quad \tilde{\gamma}^{1}(x)=t^{-q}(x) \gamma^{1}, \quad \tilde{\gamma}^{2}(x)=t^{-q}(x) \gamma^{2}, \quad \tilde{\gamma}^{3}(x)=t^{2 q-1}(x) \gamma^{3}
$$

If $\Psi(x)=t^{-1 / 2} \Psi_{0}(x)$, the equation to be solved is

$$
\left[\gamma^{0} t^{q} \partial_{t}+\gamma^{1} \partial_{x}+\gamma^{2} \partial_{y}+t^{3 q-1} \gamma^{3} \partial_{z}+t^{q} m\right] \Psi_{0}(x)=0
$$

It is a system of partial differential equations, however the fact that you have two equal and one different direction of propagation makes it difficult to separate variables. There is a general method for separating variables in the Dirac equation in curved space-time developed by Shishkin and Villalba [18]. The method consists of writing the Dirac equation in terms of the sum of two first-order differential operators

$$
\{H\} \Psi_{0}(t)=\{H\} \Gamma \Gamma^{-1} \Psi_{0}(t)=0 \Rightarrow\left(K_{i}+K_{j}\right) \Theta(x)=0, \quad\left[K_{i}, K_{j}\right]=0,
$$

where $\Theta(x)=\Gamma \Psi(x)$ is an auxiliary spinor and $\Gamma$ is a non-singular separation matrix.

We separated variables $(t, z)$ from $(x, y)$; the operators $K_{i}$ are

$$
K_{1}=t^{q}\left[\gamma^{0} \partial_{t}+t^{2 q-1} \gamma^{3} \partial_{z}+m\right] \gamma^{3} \gamma^{0}, \quad K_{2}=\left(\gamma^{1} \partial_{x}+\gamma^{2} \partial_{y}\right) \gamma^{3} \gamma^{0},
$$

where we choose $\Gamma=\gamma^{3} \gamma^{0}$. We can rewrite the Dirac equation as 


$$
\left(K_{1}+K_{2}\right) \Theta(x)=0 .
$$

As the Bianchi I model is homogeneous, it can be proposed a solution of the following form

$$
\Theta(x)=e^{i \mathbf{k} \cdot \mathbf{r}} \Theta_{0}(t)
$$

where

$$
\Theta_{0}(t)=\left(\begin{array}{l}
\Theta_{1} \\
\Theta_{2}
\end{array}\right), \quad \Theta_{1}(t)=\left(\begin{array}{l}
\xi_{1} \\
\xi_{2}
\end{array}\right), \quad \Theta_{2}(t)=\left(\begin{array}{l}
\xi_{3} \\
\xi_{4}
\end{array}\right) .
$$

By separating variables we have

$$
K_{1} \Theta_{0}=-K_{2} \Theta_{0}=-k \Theta_{0},
$$

where $k$ is a separation constant which is obtained from the equation

$$
K_{2} \Theta_{0}=i\left(\gamma^{1} k_{x}+\gamma^{2} k_{y}\right) \gamma^{3} \gamma^{0} \Theta_{0}=k \Theta_{0}
$$

We use the following representation of Dirac's matrices [23]

$$
\gamma^{0}=\left(\begin{array}{cc}
0 & -\sigma^{1} \\
\sigma^{1} & 0
\end{array}\right), \quad \gamma^{1}=\left(\begin{array}{cc}
\sigma^{1} & 0 \\
0 & -\sigma^{1}
\end{array}\right), \quad \gamma^{2}=-\left(\begin{array}{cc}
\sigma^{2} & 0 \\
0 & \sigma^{2}
\end{array}\right), \quad \gamma^{3}=\left(\begin{array}{cc}
0 & \sigma^{1} \\
\sigma^{1} & 0
\end{array}\right)
$$

The equation ( $(\overline{V .62})$ is equivalent to

$$
\begin{aligned}
& \left(k_{x}+i k_{y}\right) \Theta_{1}=k \sigma^{2} \Theta_{2} \\
& \left(k_{x}-i k_{y}\right) \sigma^{2} \Theta_{2}=k \Theta_{1} .
\end{aligned}
$$

The solution for $\Theta_{2}$ in terms of $\Theta_{1}$ is

$$
\Theta_{2}=\frac{k_{x}+i k_{y}}{k_{\perp}} \sigma^{2} \Theta_{1}
$$

where $k=\sqrt{k_{x}^{2}+k_{y}^{2}}=k_{\perp}$ and $\sigma^{2}$ is the second Pauli's matrix. Therefore the spinor $\Theta_{0}$ has the following structure

$$
\Theta_{0}=\left(\begin{array}{c}
\Theta_{1} \\
\frac{k_{x}+i k_{y}}{k_{\perp}} \sigma^{2} \Theta_{1}
\end{array}\right)
$$


To determine $\Theta_{0}$ you must solve the system of equations

$$
\begin{gathered}
\left(\frac{\mathrm{d}}{\mathrm{d} t}+i k_{z} t^{2 q-1}\right) \Theta_{1}=\sigma^{1}\left(m-i k_{\perp} t^{-q}\right) \Theta_{2} \\
\left(\frac{\mathrm{d}}{\mathrm{d} t}-i k_{z} t^{2 q-1}\right) \Theta_{2}=-\sigma^{1}\left(m+i k_{\perp} t^{-q}\right) \Theta_{1} .
\end{gathered}
$$

By decoupling the system we have an equation for the components of $\Theta_{1}(t)$

$$
\begin{aligned}
\frac{\mathrm{d}^{2} \xi_{1,2}}{\mathrm{~d} t^{2}} & \mp \frac{i q k_{\perp}}{m \mp i k_{\perp} t^{-q}} t^{-(q+1)} \frac{\mathrm{d} \xi_{1,2}}{\mathrm{~d} t}+ \\
& +\left[m^{2}+k_{\perp} t^{-2 q} \mp i k_{z}(1-2 q) t^{2 q-2}+k_{z}^{2} t^{4 q-2}+\frac{k_{\perp} k_{z} q}{m \mp i k_{\perp} t^{-q}} t^{q-2}\right] \xi_{1,2}(t)=0 .
\end{aligned}
$$

We proceed to find exact solutions from the above equation for $q=0$ and $q=1$.

\section{A. Solution for $q=0$}

For $q=0$ the equation to solve is

$$
\frac{\mathrm{d}^{2} \xi_{1,2}}{\mathrm{~d} t^{2}}+\left[m^{2}+k_{\perp}^{2}+k_{z}\left(k_{z} \mp i\right) t^{-2}\right] \xi_{1,2}(t)=0 .
$$

The solution are Bessel functions

$$
\xi_{1,2}(t)=\sqrt{t}\left[A_{1,2} H_{\nu_{\mp}}{ }^{(1)}\left(\sqrt{m^{2}+k_{\perp}^{2}} t\right)+B_{1,2} H_{\nu_{\mp}}{ }^{(2)}\left(\sqrt{m^{2}+k_{\perp}{ }^{2}} t\right)\right],
$$

where $A_{1,2}, B_{1,2}$ are integration constants and

$$
\nu_{+}=\frac{1}{2}+i k_{z}, \quad \nu_{-}=\frac{1}{2}-i k_{z} .
$$

Therefore, the spinor $\Theta_{1}(t)$ is written as

$$
\Theta_{1}(t)=\sqrt{t}\left(\begin{array}{l}
A_{1} H_{\nu_{-}}{ }^{(1)}\left(\sqrt{m^{2}+k_{\perp}{ }^{2}} t\right)+B_{1} H_{\nu_{-}}{ }^{(2)}\left(\sqrt{m^{2}+k_{\perp}{ }^{2}} t\right) \\
A_{2} H_{\nu_{+}}{ }^{(1)}\left(\sqrt{m^{2}+k_{\perp}{ }^{2}} t\right)+B_{2} H_{\nu_{+}}{ }^{(2)}\left(\sqrt{m^{2}+k_{\perp}{ }^{2}} t\right)
\end{array}\right) .
$$

The Dirac equation in the flat Kasner-type space-time was considered by Shishkin and Andrushkevic [23] and Pimentel [24] with $m=0$. 


\section{B. Solution for $q=1$}

For $q=1$, the Dirac equation has exact solutions for $m=0$; the Dirac equation for this case is

$$
\frac{\mathrm{d}^{2} \xi_{1,2}}{\mathrm{~d} t^{2}}+\frac{1}{t} \frac{\mathrm{d} \xi_{1,2}}{\mathrm{~d} t}+\left[k_{\perp}^{2} t^{-2} \pm 2 i k_{z}+k_{z}^{2} t^{2}\right] \xi_{1,2}(t)=0
$$

Introducing the new function $\xi_{1,2}(t)=t^{-1 / 2} \Xi_{1,2}(t)$, the equation is rewritten as

$$
\frac{\mathrm{d}^{2} \Xi_{1,2}}{\mathrm{~d} t^{2}}+\left[\frac{1}{t^{2}}\left(\frac{1}{4}+k_{\perp}^{2}\right) \pm 2 i k_{z}+k_{z}^{2} t^{2}\right] \Xi_{1,2}(t)=0
$$

The solution are Whittaker functions

$$
\xi_{1,2}(t)=t^{-1}\left[A_{ \pm} M_{ \pm 1 / 2, i k_{\perp} / 2}\left(i k_{z} t^{2}\right)+B_{ \pm} W_{ \pm 1 / 2, i k_{\perp} / 2}\left(i k_{z} t^{2}\right)\right] .
$$

Therefore, the spinor $\Theta_{1}(t)$ is written as

$$
\Theta_{1}(t)=t^{-1}\left(\begin{array}{c}
A_{+} M_{1 / 2, i k_{\perp} / 2}\left(i k_{z} t^{2}\right)+B_{+} W_{1 / 2, i k_{\perp} / 2}\left(i k_{z} t^{2}\right) \\
A_{-} M_{-1 / 2, i k_{\perp} / 2}\left(i k_{z} t^{2}\right)+B_{-} W_{-1 / 2, i k_{\perp} / 2}\left(i k_{z} t^{2}\right)
\end{array}\right) .
$$

\section{PRODUCTION OF SPIN 1/2 PARTICLES}

The asymptotic behaviour of the solution will define the positive and negative frequency states in $t \rightarrow 0$ and $t \rightarrow \infty$.

1. Case $q=0$

For $t \rightarrow 0$, positive frequency states expressed by a spinor $\Theta_{1(0)}^{+}(t)$ are

$$
\Theta_{1(0)}^{+}(t)=A_{(0)}^{+} \sqrt{t}\left(\begin{array}{c}
J_{\nu_{-}}\left(\sqrt{m^{2}+k_{\perp}^{2}} t\right) \\
J_{\nu_{+}}\left(\sqrt{m^{2}+k_{\perp}^{2}} t\right)
\end{array}\right)
$$

while negative frequency states are the complex conjugate of $\Theta_{1(0)}^{+}(t)$

$$
\Theta_{1(0)}^{-}(t)=A_{(0)}^{-} \sqrt{t}\left(\begin{array}{c}
J_{-\nu_{-}}\left(\sqrt{m^{2}+k_{\perp}^{2}} t\right) \\
J_{-\nu_{+}}\left(\sqrt{m^{2}+k_{\perp}^{2}} t\right)
\end{array}\right) .
$$

On the other hand, positive frequency states in $t \rightarrow \infty$ are given by

$$
\Theta_{1(\infty)}^{+}(t)=B_{(\infty)}^{+} \sqrt{t}\left(\begin{array}{l}
H_{\nu_{-}}{ }^{(2)}\left(\sqrt{m^{2}+k_{\perp}^{2}} t\right) \\
H_{\nu_{+}}{ }^{(2)}\left(\sqrt{m^{2}+k_{\perp}^{2}} t\right)
\end{array}\right)
$$


The choice of the positive and negative frequency states in was based on the asymptotic behaviour of the exact solutions ( $(\underline{V .72})$ and ( $(\mathrm{V.76})$, compared to the semiclassical behaviour (II.11), (II.14).

Using the connection formula of the Bessel functions, it is possible to express $\Theta_{1(\infty)}^{+}(t)$ in terms of $\Theta_{1(0)}^{ \pm}(t)$ with the help of the Bogolubov transformations

$$
\Theta_{1(\infty)}^{+}(t)=\alpha \Theta_{1(0)}^{+}(t)+\beta \Theta_{1(0)}^{-}(t)
$$

The Bogolubov coefficients

$$
\alpha=-\frac{i C_{(\infty)}^{+}}{D_{(0)}^{+}} \csc \left(\pi \nu_{-}\right) e^{i \pi \nu_{-}}, \quad \beta=\frac{i C_{(\infty)}^{+}}{D_{(0)}^{-}} \csc \left(\pi \nu_{-}\right)
$$

Therefore

$$
\frac{|\alpha|^{2}}{|\beta|^{2}}=e^{2 \pi k_{z}}
$$

For spinors, Bogolubov's coefficients satisfy

$$
|\alpha|^{2}-|\beta|^{2}=1
$$

The number density of created particles is

$$
n(k)=|\beta|^{2}=\left(e^{2 \pi k_{z}}+1\right)^{-1} .
$$

The number density of the created particles results in a Fermi-Dirac distribution with chemical potential $\mu_{\nu_{e}}=0$, which means that the expansion of the universe creates such particles thermally.

2. Case $q=1$ with $m=0$

The semiclassical behaviour of the $\Phi$ field without the mass term ()$m=0)$ is

$$
\Psi_{k} \sim\left\{\begin{array}{r}
e^{i \mathbf{k} \cdot \mathbf{r}} t^{ \pm i k_{\perp}}, \quad t \rightarrow 0 \\
e^{i \mathbf{k} \cdot \mathbf{r}} e^{ \pm i k_{z} t^{2} / 2}, \quad t \rightarrow \infty
\end{array}\right.
$$

For $t \rightarrow 0$, positive and negative frequency states are, respectively 


$$
\Theta_{1(0)}^{+}(t)=C_{(0)}^{+}\left(e^{i \pi}\right)^{1 / 2-\mu} t^{-1}\left(\begin{array}{c}
M_{-1 / 2,-i k_{\perp} / 2}\left(i k_{z} t^{2}\right) \\
M_{+1 / 2,-i k_{\perp} / 2}\left(i k_{z} t^{2}\right)
\end{array}\right), \quad \Theta_{1(0)}^{-}(t)=C_{(0)}^{-} t^{-1}\left(\begin{array}{c}
M_{1 / 2, i k_{\perp} / 2}\left(i k_{z} t^{2}\right) \\
M_{-1 / 2, i k_{\perp} / 2}\left(i k_{z} t^{2}\right)
\end{array}\right)
$$

while for $t \rightarrow \infty$ positive frequency states are

$$
\Theta_{1(\infty)}^{+}(t)=D_{(\infty)}^{+} t^{-1}\left(\begin{array}{c}
W_{1 / 2, i k_{\perp} / 2}\left(i k_{z} t^{2}\right) \\
W_{-1 / 2, i k_{\perp} / 2}\left(i k_{z} t^{2}\right)
\end{array}\right)
$$

The spinors $\Theta_{1(\infty)}^{+}(t), \Theta_{1(0)}^{-}(t)$ are related through Bogolubov's transformations

$$
\Theta_{1(\infty)}^{+}(t)=\alpha \Theta_{1(0)}^{+}(t)+\beta \Theta_{1(0)}^{-}(t)
$$

Using the connection ratio of the Whittaker functions the following coefficients are obtained

$$
\alpha=\frac{D_{(\infty)}^{+}}{C_{(0)}^{+}} \frac{\Gamma\left(-i k_{z}\right)}{\Gamma\left(-i k_{z} / 2\right)} e^{\pi k_{\perp} / 2} e^{-i \pi / 2}, \quad \beta=\frac{D_{(\infty)}}{C_{(0)}^{-}} \frac{\Gamma\left(i k_{z}\right)}{\Gamma\left(i k_{z} / 2\right)}
$$

Therefore

$$
\frac{|\alpha|^{2}}{|\beta|^{2}}=e^{\pi k_{\perp}}
$$

The number density of the created particles obeys the Fermi-Dirac distribution.

$$
n(k)=|\beta|^{2}=\left(\frac{|\alpha|^{2}}{|\beta|^{2}}+1\right)^{-1}=\left(e^{\pi k_{\perp}}+1\right)^{-1}
$$

\section{FINAL REMARKS}

In this article we discuss creation of scalar and spin $1 / 2$ particles in some LRS Bianchi I models using the semi-classical method to identify and define the positive and negative frequency states of the fields at the asymptotic limits of the $t \rightarrow 0, t \rightarrow \infty$ expansion. For scalar particles, the same results were obtained as those by Duru with the path integral method, while for massless spin $1 / 2$ particles the result obtained is the Fermi-Dirac distribution. This calculation corroborates that the semi-classical method coincides with the more sophisticated of path integrals to define the vacuum state in general relativity, which makes it efficient and accessible in many cases of interest. 


\section{ACKNOWLEGEMENTS}

This work was partially supported by PRODEP UAM-I-CA-43 and F. P. by CONACYT grant 706699 .

[1] E. Schrödinger, Physica 6, 899, (1939).

[2] S. W. Hawking, Commun.Math. Phys. 43, 199-220 (1975).

[3] L. Parker, Phys. Rev. Lett. 21, 562 (1968).

[4] L. Parker, Phys. Rev. 183, 1057 (1969).

[5] L. Parker, Phys. Rev. D 3, 346 (1971).

[6] L. Parker, The production of elementary particles by strong gravitational fields, pp. 107-227 in Asymptotic structure of space-time, edited by F. P. Esposito and L. Witten, Plenum Press N.Y. (1977).

[7] L. Parker, Nature 261, 20 (1976).

[8] V. M. Villalba, Phys. Rev. 52, 3742 (1995).

[9] A. A. Grib, S. G. Mamayev, V. M. Mostepanenko, Gen. Relat. Grav. 7, 535-547 (1976).

[10] J. Audretsch, G. Schafer, J. Phys. A. 11, 1583 (1978).

[11] S. Moradi, J. Geom. Phys. 59, 173-184 (2009).

[12] I. Bukhbinder, Sov. Phys. J. 23, 545-548 (1980).

[13] D. M. Chitre, J. Hartle, Phys. Rev. D. 16, 251 (1977).

[14] I. Duru, Gen. Relat. Grav. 26, 969 (1994).

[15] V. N. Villalba, J. Theor. Phys. 36, 1321 (1997).

[16] V. M. Villalba, W. Greiner, Phys. Rev. D. 65, 025007 (2001).

[17] K. C. Jacobs, Astro. J. 153, 661 (1968).

[18] G. V. Shishkin, V. M. Villalba, J. Math. Phys. 30, 2132 (1989).

[19] L. O. Pimentel, Gen. Relat. Grav. 24, 985 (1992).

[20] F. W. Olver, D. W. Lozier, R. F. Boisvert, C. W. Clark, NIST Handbook of Mathematical Functions, Cambridge University press, New York, NY, (2010).

[21] N. D. Birrel and P. C. Davis, Quantum Fields in Curved Space, Cambridge University Press, Cambridge (1982).

[22] P. Collas, D. Klein, The Dirac Equation in Curved Spacetime, a Guide for Calculations Springer, Berlin, (2019); arXiv preprint :1809.02764[gr-qc].

[23] G. V. Shishkin, I. E. Andrushkevich, Phys. Lett. A 110, 84 (1985).

[24] L. O. Pimentel, Int. J. Theor. Phys. 32, 979 (1993). 